\title{
Temporal Lobe Epilepsy after Refractory Status Epilepticus: An Illustrative Case and Review of the Literature
}

\author{
J. Gordon Boyd, ${ }^{1}$ Derek B. Debicki, ${ }^{2}$ and G. Bryan Young ${ }^{2}$ \\ ${ }^{1}$ Division of Critical Care Medicine, Kingston General Hospital, Queen's University, Kingston, ON, Canada K7L 2V7 \\ ${ }^{2}$ Department of Clinical Neurological Sciences, London Health Sciences Centre, London, ON, Canada N6A 5A5
}

Correspondence should be addressed to G. Bryan Young, bryan.young@lhsc.on.ca

Received 4 October 2011; Accepted 29 January 2012

Academic Editor: Seyed M. Mirsattari

Copyright ( 92012 J. Gordon Boyd et al. This is an open access article distributed under the Creative Commons Attribution License, which permits unrestricted use, distribution, and reproduction in any medium, provided the original work is properly cited.

New onset refractory status epilepticus (NORSE) is a relatively newly defined disease entity, where otherwise healthy individuals develop unrelenting seizures that do not respond to conventional anticonvulsant therapy and may require months of therapy with anesthetic drugs. We have described a case of NORSE who subsequently developed mesial temporal lobe sclerosis (MTS) and recurrent temporal lobe seizures. We discuss the possible pathophysiological mechanisms by which refractory seizures may contribute to the development of temporal lobe epilepsy (TLE).

\section{Introduction}

Temporal lobe pathology is associated with critical illness via a variety of mechanisms. Cardiac arrhythmias and asystole are known to complicate temporal lobe seizures [1]. Severe infections of the nervous system, such as herpes simplex virus infection, can cause hemorrhagic destruction of the temporal lobe and subsequent cerebral edema and herniation. The temporal tips and mesial temporal lobe structures are also commonly affected in traumatic brain injury, often resulting in memory impairment, posttraumatic epilepsy, and other deficits [2-4]. The hippocampus is the most vulnerable structure in cases of hypoxic-ischemic insults, as with transient cardiac arrest, with profound memory deficit as a frequent outcome [5]. Perhaps one of the most common reasons that an intensivist (or neurointensivist) may come to care for patients with ultimate temporal lobe dysfunction is with seizures, particularly status epilepticus.

Status epilepticus has often been associated with temporal lobe epilepsy as being a complication of, or a cause of, temporal lobe pathology. Although there have been many studies which have evaluated the clinical outcome of patients with status epilepticus (and refractory status epilepticus) in the intensive care setting [6-11], there is relatively little data regarding temporal lobe epilepsy as a sequela of prolonged seizure activity outside of febrile status epilepticus [10-13]. More importantly, the exact mechanisms relating an episode of status epilepticus with subsequent temporal lobe epilepsy remain largely unclear.

In this report, we provide a case illustration of a previously well patient who subsequently developed multifocal epilepsy following new-onset refractory status epilepticus (NORSE) syndrome of unclear etiology [14]. The epilepsy syndrome was characterized by independent bilateral temporal lobe epilepsy (TLE), plus multifocal interictal epileptic spikes that were also maximal in the temporal regions. We use this case to illustrate that refractory status epilepticus can be associated with subsequent temporal lobe pathology, including MTS, and as a result can lead to an ongoing temporal lobe seizure disorder. The literature linking status epilepticus with TLE is reviewed.

\section{Case Report}

This previously healthy 22-year-old right-handed woman presented with generalized tonic-clonic seizures on the background of a 3-day history of fever, malaise, and a reddish skin lesion on her posterior calf. This occurred shortly after her return from travelling to the northeastern United States. She continued to have seizures despite being treated with 
midazolam and phenytoin; therefore she was intubated for airway protection, started on propofol, and placed on continuous EEG monitoring. Her antimicrobial therapy included sulfamethoxazole/trimethoprim for the skin lesion, as well as ceftriaxone and acyclovir as empiric coverage for meningoencephalitis. She continued to have breakthrough seizures, despite multiple anticonvulsant medications, including levetiracetam, ketamine, isofluorane, phenobarbital, pregabalin, and valproic acid. An exhaustive search for infectious, autoimmune, neoplastic, and paraneoplastic causes of refractory seizures did not yield any specific etiology (Table 1). She was treated with two empiric courses of intravenous immunoglobulin for a presumed autoimmune/ paraneoplastic limbic encephalitis. A typical seizure during her acute illness is shown in Figure 1. The seizure originated from the left frontal/anterior temporal region and remained relatively focal. At the same time, there are independent, rhythmical, and sharply formed theta waves in the right frontal region (Figures 1(a)-1(c)).

The patient's seizures eventually ceased, and she could be weaned off the anaesthetic agents and ventilatory support. After over two months of refractory seizures, she was stable enough to be transferred to the neurology ward. At that time, she was oriented to person, place, and time and was able to follow one-step commands. She had moderate-to-severe proximal muscle weakness, likely on the basis of critical illness myopathy. She had gaze-evoked nystagmus and a symmetrical action tremor, presumably due to the effects of anticonvulsant medications. Five months after the onset of her refractory status epilepticus, she had returned home from the rehabilitation centre to live with her parents and was able to work part time. She had ongoing difficulties with frequent simple partial, complex partial (with staring and automatisms) and secondarily generalized seizures, despite being on five anticonvulsant medications (clobazam, phenytoin, levetiracetam, topiramate, and pregabalin). Followup neuropsychological testing demonstrated moderately-severely impaired verbal and visual memory, an IQ in the low averageaverage range, and moderately impaired language functioning. The possibility of medication effects on her neuropsychological testing could not be fully excluded.

She had serial MR imaging throughout her hospital stay and clinical followup. At the onset of status epilepticus, the MRI was completely normal (not shown). Ten days after the onset of refractory status epilepticus, her hippocampi were edematous and had increased T2 signal intensity (Figure 2(a)). Subsequent MRI studies performed at 1 (Figure 2(b)), 2 (Figure 2(c)), and 6 (Figure 2(d)) months later demonstrated progressive hippocampal atrophy and mild decrease in T2 signal intensity.

The case illustrates the potential relationship between refractory status epilepticus and TLE. This relationship can be complex and may involve ongoing focal and/or generalized seizures from a symptomatic brain lesion. Moreover, it may be associated with the development of MTS, regardless whether an underlying pathologic lesion is identified or not, as in the case presented above. In the following, we review the literature as it relates to the association between nonfebrile status epilepticus, temporal lobe pathology, and temporal lobe epilepsy.
TABLE 1

\begin{tabular}{ll}
\hline Diagnostic category & Negative laboratory tests \\
\hline $\begin{array}{l}\text { Blood/serology: parainfluenza virus, } \\
\text { adenovirus, rhinovirus, respiratory } \\
\text { syncytial virus, HIV, flava cirus, west } \\
\text { nile virus, herpes simplex virus, } \\
\text { cytomegalovirus, lyme, California } \\
\text { equine encephalitis (initially equivocal, } \\
\text { then negative on subsequent testing) } \\
\text { Cerebrospinal fluid: syphilis, St. Louis } \\
\text { encephalopathy, powassan, } \\
\text { arboviruses, enterovirus, Chlamydia, } \\
\text { mycoplasma, influenza (A and B), } \\
\text { varicella zoster virus }\end{array}$ \\
Infectious & $\begin{array}{l}\text { ANA, ENA, ANCA, } \\
\text { antithyroperoxidase }\end{array}$ \\
Autoimmune & $\begin{array}{l}\text { Antivoltage-gated potassium channels, } \\
\text { antivoltage-gated calcium channels, } \\
\text { anti-NMDA (NR-1) }\end{array}$ \\
\hline
\end{tabular}

Multiple case series have reported the mortality and morbidity of patients with status epilepticus in the intensive care setting [6-11]. For example, mortality has been reported to range between $7 \%$ and $39 \%$, while morbidity, including ongoing seizures, is reported to range between $3-13 \%$ in patients with status epilepticus (e.g., [10]). These rates are increased if seizure activity is prolonged and refractory to medical treatment, although even in these cases long term survival and some functional recovery are possible [9].

It has been recognized that symptomatic and unprovoked epilepsy may follow as a consequence of status epilepticus, particularly if it is refractory in nature [10-13]. However, the extent to which status epilepticus itself contributes to the process of epileptogenesis remains unclear. In part, this is because the inciting cause for the episode of status epilepticus can often itself be a risk factor for ongoing seizures [15]. For example, acute symptomatic seizures may occur as a result of traumatic brain injury, central nervous system infections, cerebrovascular disease, brain tumours, neurosurgery, and neurodegenerative disorders [13]. These lesions increase the risk for the development of subsequent seizures and this risk appears to be increased if it is related to an episode of status epilepticus. This suggests a synergistic effect of epileptogenic potential in the cases of symptomatic status epilepticus with defined lesions as a precipitating etiology [13]. Moreover, symptomatic status epilepticus may be related to the development of MTS, which itself can lead to TLE. Therefore, a situation may arise where TLE associated with MTS may overlap seizure activity related to the initial lesion itself (i.e., so called dual pathology) [16]. Although there have been many reported cases suggesting that status epilepticus leads to MTS, a direct link is difficult to establish, particularly if a lesion is known to be the underlying pathology.

One of the first descriptions of progressive radiological changes in the hippocampus related to status epilepticus in an adult was by Wieshmann et al. [17]. These authors described a case report of a 30-year-old woman with status 


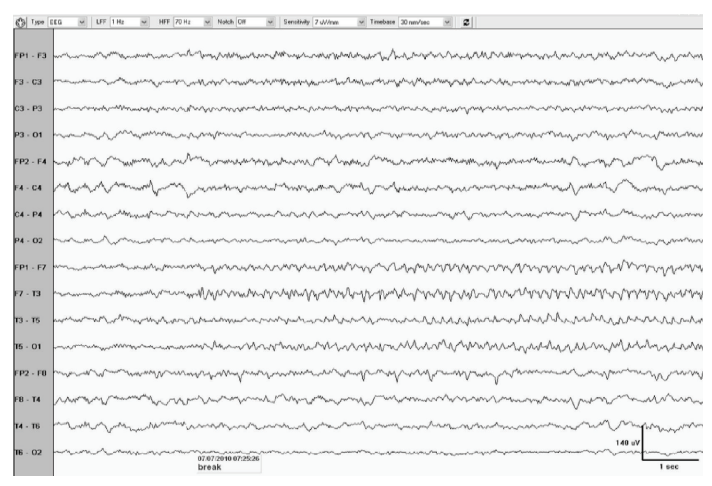

(a)

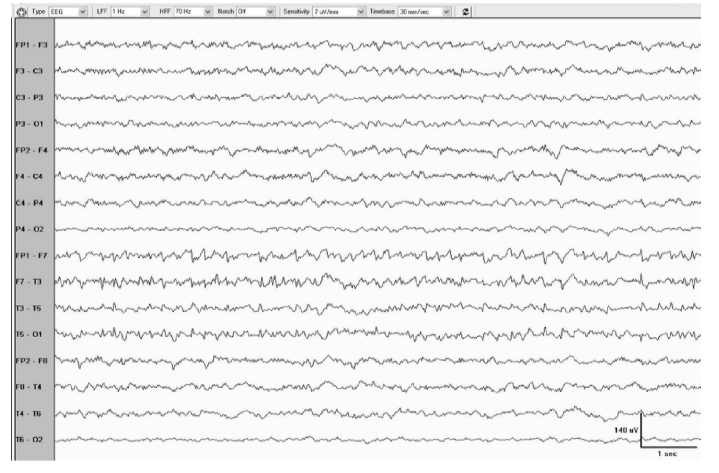

(b)

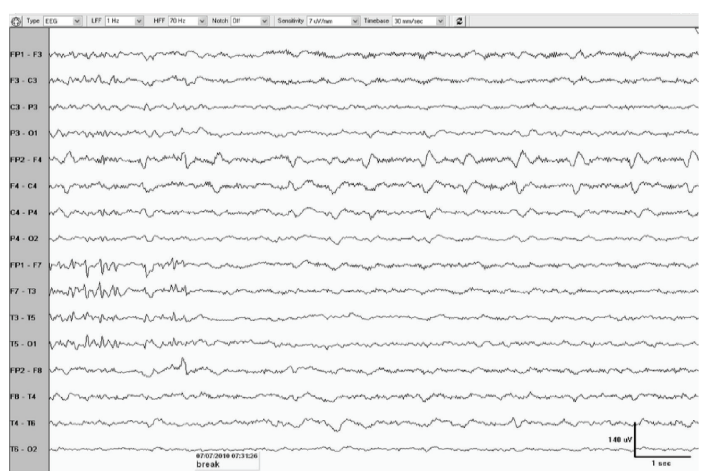

(c)

FIGURE 1: Typical electrographic seizure. This anterior-posterior bipolar montage contains an electrographic seizure which originates in the left frontal/anterior temporal region. The seizure does not generalize to the right hemisphere. Independent sharply formed slow waves are seen in the right frontal-central region. These sharply formed slow waves become semirhythmical but do not evolve into an electrographic seizure during the epoch shown. The EEG is calibrated to $7 \mathrm{~V} / \mathrm{mm}$, and the paper speed is $30 \mathrm{~mm} /$ second. The low frequency filter is set for $1 \mathrm{~Hz}$, and the high frequency filter is set at $70 \mathrm{~Hz}$. The $60 \mathrm{~Hz}$ notch filter is off.

epilepticus due to herpes simplex encephalitis. Although she survived the initial illness, she developed severe memory problems and recurrent focal seizures with secondary generalization, which were refractory to medical therapy. The authors clearly document progressive atrophy in both hippocampi over a 5-year period. Although the atrophy may be attributed to the initial event of status epilepticus, this hippocampal atrophy may also have been related to the herpes simplex infection or the ongoing, medically refractory seizures [17]. More recently, there have been published longitudinal assessments of hippocampal volume and progressive MTS $[18,19]$. However, in these cases, the causality of MTS from status epilepticus could not be confidently defined as there was likely underlying structural pathology present prior to the onset of seizures. Likewise, in longitudinal structural imaging in patients with febrile status epilepticus [20-22], the ongoing debate regarding the development of MTS following, in these cases, febrile convulsive status epilepticus, is that there still may be preexisting structural or genetic substrates that predispose these children to developing both febrile seizures and MTS [23].

With regards to our case, while we cannot eliminate the possibility of an underlying genetic predisposition to refractory seizures, status epilepticus, and MTS, our radiological evaluation did not reveal any underlying substrate for seizures (i.e., malformations of cortical development). We would argue that in our case much of the neuronal loss and subsequent gliosis within the hippocampus was due to a direct effect of the seizures themselves, as has been previously demonstrated in animal models of status epilepticus [24]. A similar argument was put forth following a description of an adult patient with refractory convulsive status epilepticus, also of unknown etiology. At presentation, the patient had a documented unremarkable MRI, which developed into left MTS, as demonstrated on follow-up MRI 111 days later. At that point the patient continued to require medically induced electrographic suppression of ongoing focal and generalized seizures [25]. In a comparable clinico-pathological description of refractory status epilepticus in a 3-year-old boy, initial imaging was completely normal; however, progressive T2 hyperintensity and hippocampal atrophy occurred during his 6-week course of refractory status epilepticus that occurred in the context of a febrile illness [26]. The neuropathological assessment demonstrated hippocampal neuronal loss and gliosis, in the absence of any other congenital lesion. Although these individual cases lend support to the idea that status epilepticus initiates or participates in the epileptogenesis of temporal lobe seizures, a clear definitive 


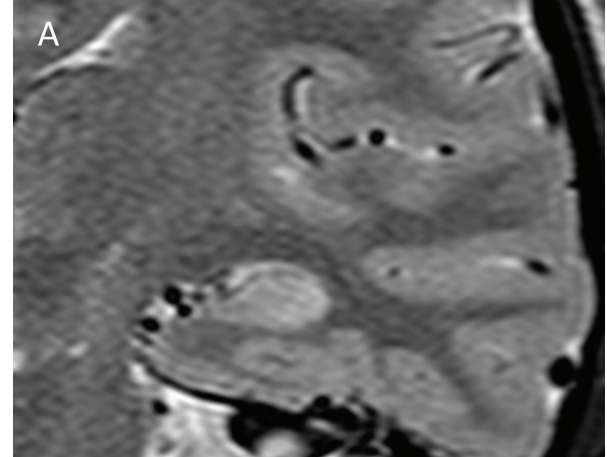

(a)

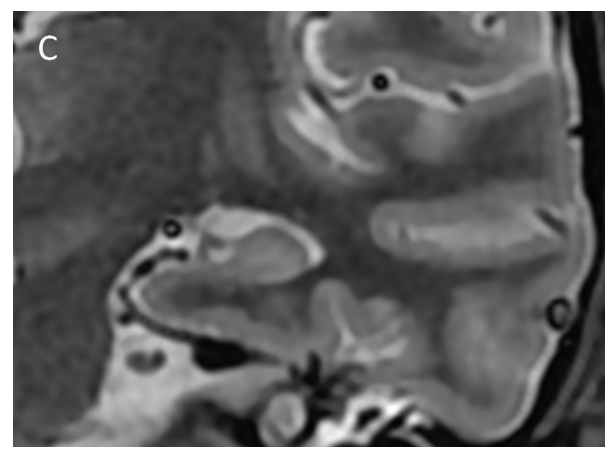

(c)

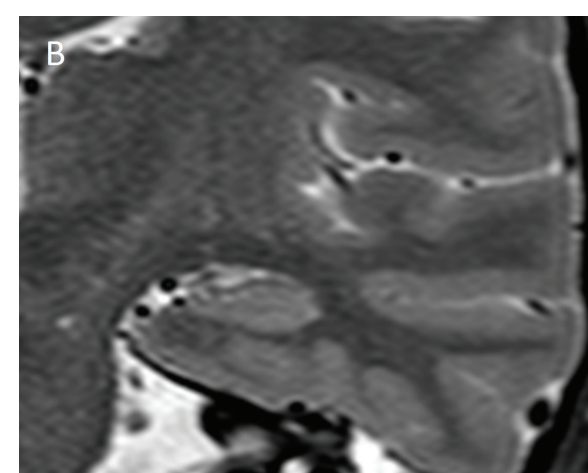

(b)

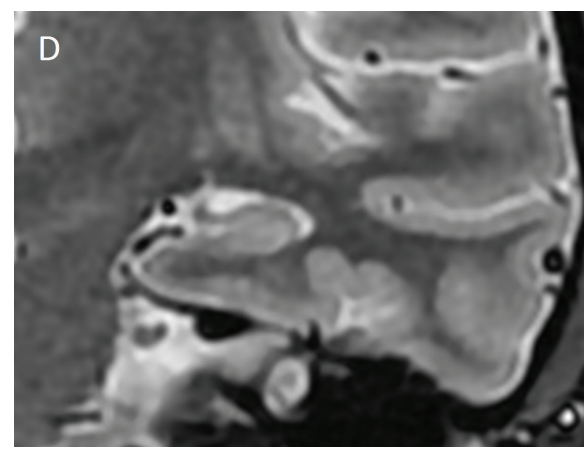

(d)

FIgure 2: Longitudinal MRI, Coronal T2 (A, B), and fluid inversion recovery (FIR; C, D) images are shown through the hippocampus for the patient described in case number 2. While the initial imaging for this patient was normal (not shown), the hippocampus appears bright and somewhat edematous 10 days after the onset of status epilepticus (A). Thirty days after the onset of refractory status epilepticus (B), the edema has resolved, and the hippocampus appears atrophic. At 2 (C) and 6 (D) months after the onset of status epilepticus, progressive hippocampal atrophy is noted.

link has not yet been established. More importantly, the mechanism by which this could occur is still not clearly understood.

One suggestion has been that inflammation may play a key role in epileptogenesis following prolonged seizure activity. For example, in prolonged febrile seizures in children, it has been proposed that the inflammatory response during these episodes may contribute to the development of MTS, which is often associated with ongoing TLE in these patients [27]. Similarly, in adults without a previous history of epilepsy, it has become increasingly recognized that inflammation [28] and blood-brain barrier dysfunction (e.g., [29]) resulting from seizure activity might ultimately lead to cell loss and contribute to the development of hyperexcitable circuits. Indeed, inflammation within the brain can be induced by mechanisms such as trauma, infection, vascular pathology or other structural brain lesions, but the balance between the destructive and neuroprotective roles of inflammation in these scenarios is not fully understood [28]. Disorders such as lupus, vasculitis, paraneoplastic limbic encephalitis, anti$\mathrm{N}$-methyl-D-aspartate (anti-NMDA) receptor antibody syndrome, and antivoltage-gated potassium channel (antiVGKC) antibody syndrome, all of which can lead to the manifestation of seizures and status epilepticus, are due to immune-mediated mechanisms [28] and are treated with therapies (i.e., IVIg, plasmapheresis, and corticosteroids) targeted against the immune response.

Therefore, autoimmune processes and the inflammatory response may not only cause seizures (i.e., such as in limbic encephalitis), but may subsequently contribute to the perpetuation of seizures during status epilepticus and may participate in the epileptogenesis of future seizures from focal lesions or from the development of MTS. In a recent retrospective study of 38 patients with adult onset TLE and hippocampal sclerosis, more than half was associated with diagnosed or suspected limbic encephalitis (including paraneoplastic and anti-VGKC antibody syndrome). The remaining either had a clear precipitating lesion with subsequent hippocampal atrophy (i.e., dual pathology) or were idiopathic [16]. This highlights the relationship between new-onset seizures in the context of immune-mediated processes or discrete brain lesions and the development of TLE associated with MTS. Further work is required to help determine the exact role of the inflammatory response in the development of TLE following status epilepticus. This will help to clarify the mechanisms underlying these complex relationships, particularly since not all patients who have an episode of status epilepticus develop ongoing epilepsy and/or MTS. More importantly, it may help to define more clearly therapeutic targets in cases of refractory status epilepticus. 


\section{Summary and Conclusions}

Temporal lobe pathology can be associated with critical illness in a number of ways, most commonly in the form of seizures, particularly status epilepticus and the subsequent development of TLE. The relationship between status epilepticus and TLE can be seemingly complex. This is because it may involve the development of MTS leading to seizures of temporal lobe origin. However, this can be complicated by recurrent seizures arising from the focus of initial structural pathology, if it can be identified. The case presented above illustrates the development of MTS and ongoing seizures in the context of a previously healthy young adult with a newonset refractory status epilepticus syndrome of unknown etiology. It has been proposed that ongoing inflammation might contribute to the mechanisms that underlie complications associated with status epilepticus, including a refractory state and the development of MTS. Further work is required to demonstrate more clearly the relationship between status epilepticus and TLE. In addition, more research is necessary to help develop strategies for treating patients with status epilepticus which becomes refractory to the medications and therapeutic targets currently used in the intensive care setting.

\section{Acknowledgment}

The authors would like to thank the EEG technologists whose role in facilitating continuous EEG monitoring cannot be underestimated.

\section{References}

[1] D. Sorajja, M. D. Bhakta, J. F. Drazkowski, and G. T. Altemose, "Ictal asystole," Heart Rhythm, vol. 8, no. 2, pp. 331-332, 2011.

[2] N. R. Temkin, "Preventing and treating posttraumatic seizures: the human experience," Epilepsia, vol. 50, no. 2, supplement, pp. 10-13, 2009.

[3] S. Riggio and M. Wong, "Neurobehavioral sequelae of traumatic brain injury," Mount Sinai Journal of Medicine, vol. 76, no. 2, pp. 163-172, 2009.

[4] P. Azouvi, C. Vallat-Azouvi, and A. Belmont, "Cognitive deficits after traumatic coma," Progress in Brain Research, vol. 177, no. C, pp. 89-110, 2009.

[5] V. R. M. P. Moulaert, J. A. Verbunt, C. M. van Heugten, and D. T. Wade, "Cognitive impairments in survivors of out-of-hospital cardiac arrest: a systematic review," Resuscitation, vol. 80, no. 3, pp. 297-305, 2009.

[6] J. M. Oxbury and C. W. M. Whitty, "Causes and consequences of status epilepticus in adults: a study of 86 cases," Brain, vol. 94, no. 4, pp. 733-744, 1971.

[7] J. Aicardi and J. J. Chevrie, "Convulsive status epilepticus in infants and children. A study of 239 cases," Epilepsia, vol. 11, no. 2, pp. 187-197, 1970.

[8] G. D. Cascino, D. Hesdorffer, G. Logroscino, and W. A. Hauser, "Morbidity of nonfebrile status epilepticus in Rochester, Minnesota, 1965-1984," Epilepsia, vol. 39, no. 8, pp. 829-832, 1998.

[9] A. D. Cooper, J. W. Britton, and A. A. Rabinstein, "Functional and cognitive outcome in prolonged refractory status epilepticus," Archives of Neurology, vol. 66, no. 12, pp. 1505-1509, 2009.
[10] M. Holtkamp, J. Othman, K. Buchheim, and H. Meierkord, "Predictors and prognosis of refractory status epilepticus treated in a neurological intensive care unit," Journal of Neurology, Neurosurgery and Psychiatry, vol. 76, no. 4, pp. 534-539, 2005.

[11] A. Neligan and S. D. Shorvon, "Prognostic factors, morbidity and mortality in tonic-clonic status epilepticus: a review," Epilepsy Research, vol. 93, no. 1, pp. 1-10, 2011.

[12] D. C. Hesdorffer, G. Logroscino, G. Cascino, J. F. Annegers, and W. A. Hauser, "Incidence of status epilepticus in Rochester, Minnesota, 1965-1984," Neurology, vol. 50, no. 3, pp. 735$741,1998$.

[13] D. C. Hesdorffer, G. Logroscino, G. Cascino, J. F. Annegers, and W. A. Hauser, "Risk of unprovoked seizure after acute symptomatic seizure: effect of status epilepticus," Annals of Neurology, vol. 44, no. 6, pp. 908-912, 1998.

[14] D. J. Costello, R. D. Kilbride, and A. J. Cole, "Cryptogenic new onset refractory status epilepticus (NORSE) in adults-Infectious or not?" Journal of the Neurological Sciences, vol. 277, no. 1-2, pp. 26-31, 2009.

[15] S. T. Herman, "Epilepsy after brain insult: targeting epileptogenesis," Neurology, vol. 59, no. 9, supplement, pp. S21-S26, 2002.

[16] C. G. Bien, H. Urbach, J. Schramm et al., "Limbic encephalitis as a precipitating event in adult-onset temporal lobe epilepsy," Neurology, vol. 69, no. 12, pp. 1236-1244, 2007.

[17] U. C. Wieshmann, F. G. Woermann, L. Lemieux et al., "Development of hippocampal atrophy: a serial magnetic resonance imaging study in a patient who developed epilepsy after generalized status epilepticus," Epilepsia, vol. 38, no. 11, pp. 1238-1241, 1997.

[18] G. Gong, F. Shi, L. Concha, C. Beaulieu, and D. W. Gross, "Insights into the sequence of structural consequences of convulsive status epilepticus: a longitudinal MRI study," Epilepsia, vol. 49, no. 11, pp. 1941-1945, 2008.

[19] P. M. Vespa, D. L. McArthur, Y. Xu et al., "Nonconvulsive seizures after traumatic brain injury are associated with hippocampal atrophy," Neurology, vol. 75, no. 9, pp. 792-798, 2010.

[20] R. C. Scott, D. G. Gadian, M. D. King et al., "Magnetic resonance imaging findings within 5 days of status epilepticus in childhood," Brain, vol. 125, no. 9, pp. 1951-1959, 2002.

[21] R. C. Scott, M. D. King, D. G. Gadian, B. G. R. Neville, and A. Connelly, "Hippocampal abnormalities after prolonged febrile convulsion: a longitudinal MRI study," Brain, vol. 126, no. 11, pp. 2551-2557, 2003.

[22] J. M. Provenzale, D. P. Barboriak, K. VanLandingham, J. MacFall, D. Delong, and D. V. Lewis, "Hippocampal MRI signal hyperintensity after febrile status epilepticus is predictive of subsequent mesial temporal sclerosis," American Journal of Roentgenology, vol. 190, no. 4, pp. 976-983, 2008.

[23] F. Cendes, "Febrile seizures and mesial temporal sclerosis," Current Opinion in Neurology, vol. 17, no. 2, pp. 161-164, 2004.

[24] F. A. Scorza, R. M. Arida, M. G. Naffah-Mazzacoratti, D. A. Scerni, L. Calderazzo, and E. A. Cavalheiro, "The pilocarpine model of epilepsy: what have we learned?" Anais da Academia Brasileira de Ciencias, vol. 81, no. 3, pp. 345-365, 2009.

[25] K. Standley, R. Abdulmassih, and S. Benbadis, "Good outcome is possible after months of refractory status epilepticus: lesson learned," Epilepsia, vol. 53, no. 1, pp. e17-e20, 2012.

[26] C. E. Stafstrom, R. D. Tien, T. J. Montine, and R. M. Boustany, "Refractory status epilepticus associated with progressive magnetic resonance imaging signal change and hippocampal 
neuronal loss," Journal of Epilepsy, vol. 9, no. 4, pp. 253-258, 1996.

[27] S. McClelland, C. M. Dubé, J. Yang, and T. Z. Baram, "Epileptogenesis after prolonged febrile seizures: mechanisms, biomarkers and therapeutic opportunities," Neuroscience Letters, vol. 497, no. 3, pp. 155-162, 2011.

[28] A. Vezzani, J. French, T. Bartfai, and T. Z. Baram, "The role of inflammation in epilepsy," Nature Reviews Neurology, vol. 7, no. 1, pp. 31-40, 2011.

[29] A. Friedman, "Blood-brain barrier dysfunction, status epilepticus, seizures, and epilepsy: a puzzle of a chicken and egg?" Epilepsia, vol. 52, no. 8, supplement, pp. 19-20, 2011. 


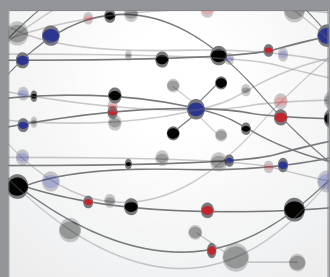

The Scientific World Journal
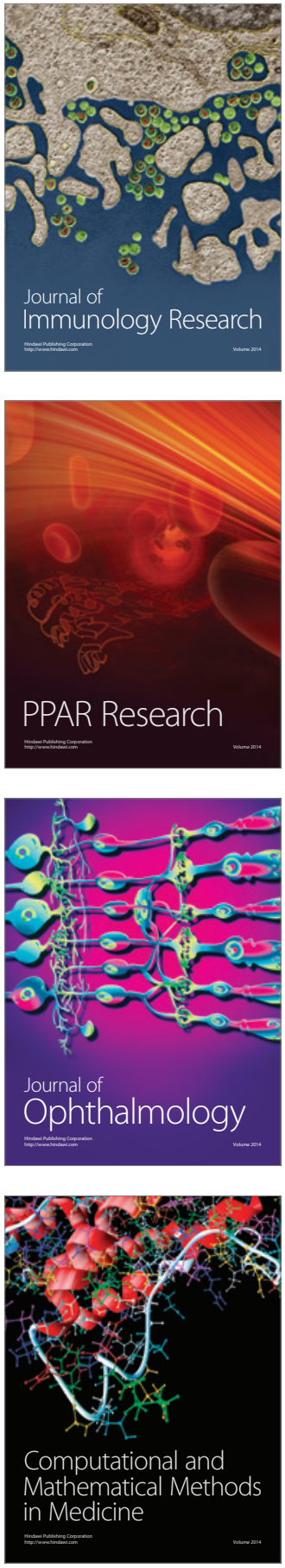

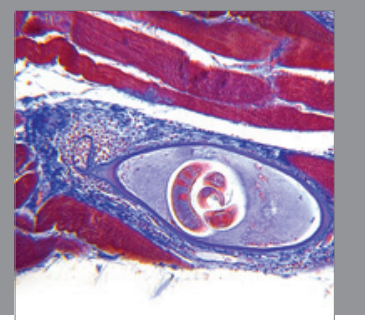

Gastroenterology

Research and Practice
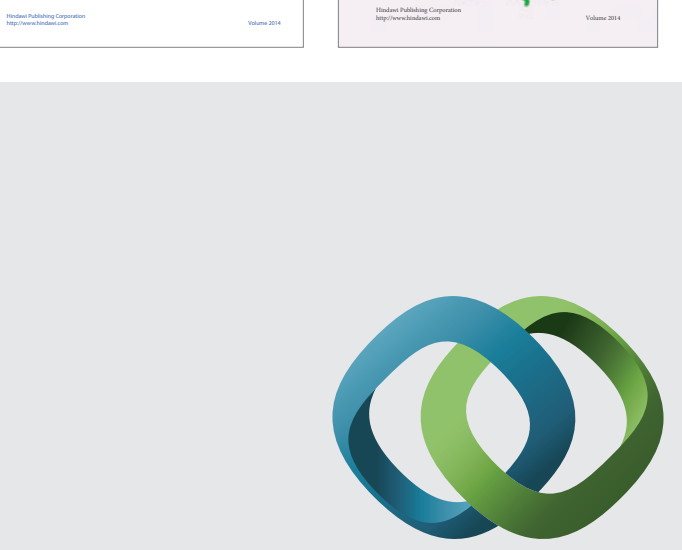

\section{Hindawi}

Submit your manuscripts at

http://www.hindawi.com
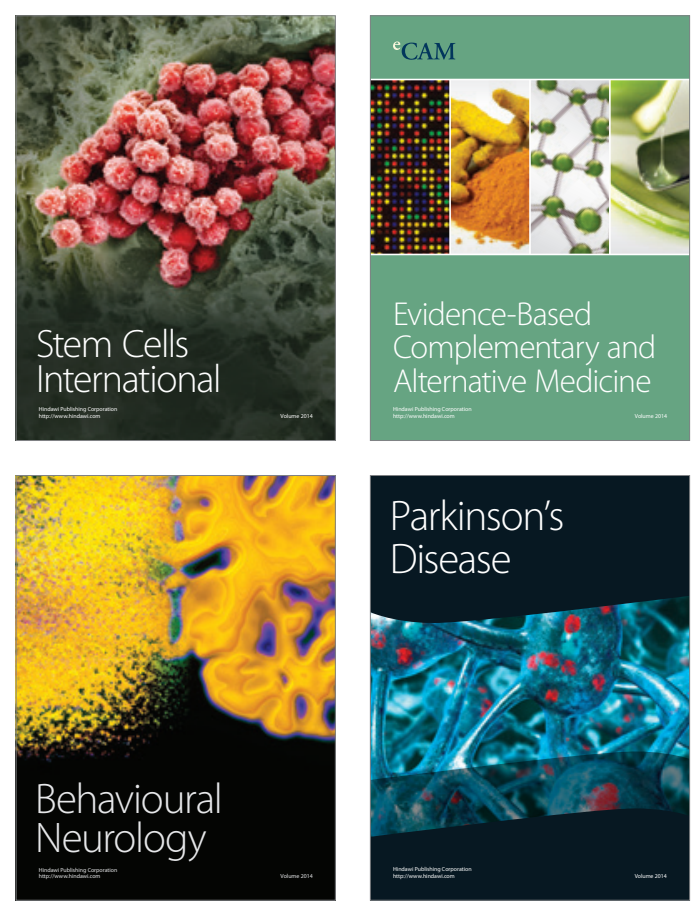

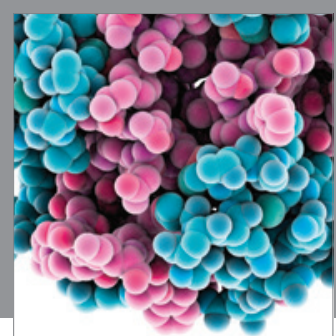

Journal of
Diabetes Research

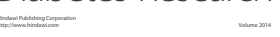

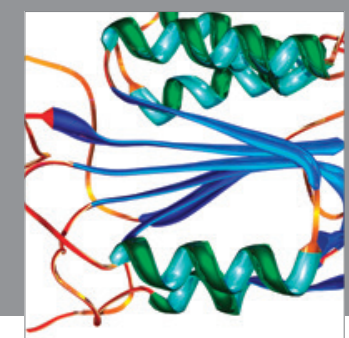

Disease Markers
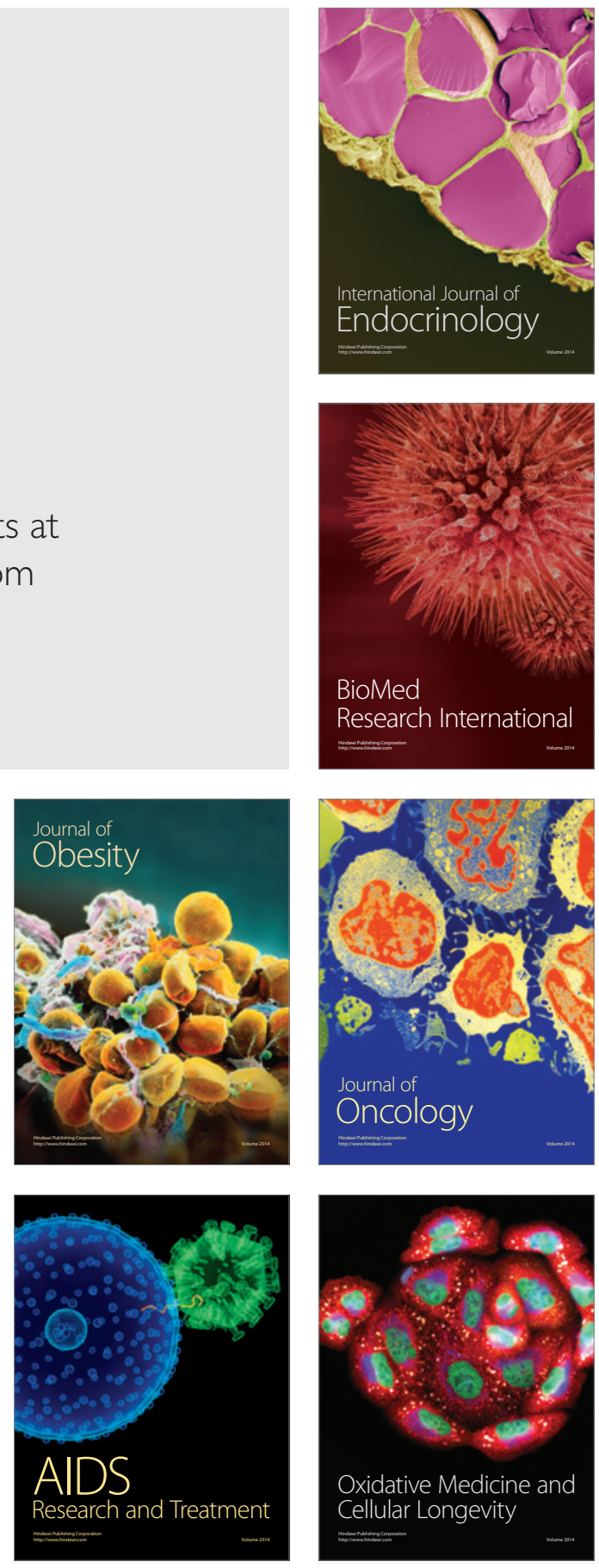